\title{
Switching between aflibercept and ranibizumab in the treatment of refractory exudative age-related macular degeneration
}

\author{
Małgorzata Wichrowska, Jarosław Kocięcki, Wojciech Suda
}

Department of Ophthalmology, Poznan University of Medical Sciences Head: Prof. Jarosław Kocięcki, MD, PhD

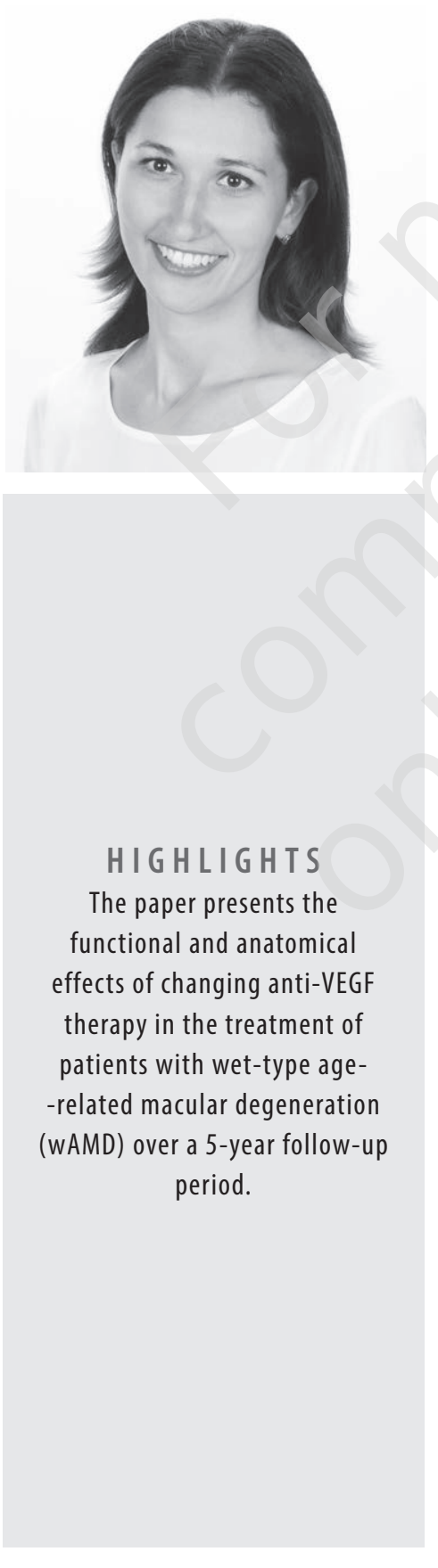

\section{ABSTRACT}

Introduction: Aflibercept and ranibizumab have become the most used drugs in the treatment of wet age-related macular degeneration. Some patients; however, do not respond to aflibercept and ranibizumab treatment. Our aim was to determine whether the switch from aflibercept to ranibizumab, and then switchback, is effective in a group of patients poorly responding to aflibercept. We assessed best corrected visual acuity, central retinal thickness, and the presence of intra- and subretinal fluid (respectively).

Material and methods: We conducted a retrospective comparative case series study. Twenty eyes of 20 patients, who had no inhibition of disease activity after at least one year of treatment with aflibercept, were included in the study. If after switch to ranibizumab the response to the drug was again inadequate, we returned to aflibercept. We divided the study population into two groups: treatment-naïve and patients who had received injections before entering the Drug Program for the Treatment of Age-Related Exudative Macular Degeneration.

Results: We observed an improvement in both best corrected visual acuity and central retinal thickness parameters, but only in the treatment-naïve group. We did not observe any statistically credible effects for sub- and intraretinal fluid in the studied groups.

Conclusions: Drug conversion may be beneficial for patients poorly responding to primary therapy. Greater improvement is seen in previously untreated patients.

Key words: anti-VEGF therapy, AMD, therapy switch 


\section{INTRODUCTION}

The availability of intravitreal anti-VEGF drugs has revolutionized the treatment of age-related macular degeneration (wAMD), becoming the gold standard in many countries [1].

The anti-VEGF drugs inhibit the development of the neovascular membrane in the macular region, reduce the central retinal thickness (CRT) by decreasing the amount of sub- and intraretinal fluid. The action of these drugs allows patients to maintain a useful visual acuity (VA). The most commonly used drugs in this group of patients are aflibercept, ranibizumab and bevacizumab, with the last one used off-label $[1,2]$. Data from the literature show that the treatment efficacy of all preparations seems to be undeniable and comparable [3, 4]. In clinical practice; however, we encounter patients whose response to treatment is insufficient and we cannot achieve lack of sub- or intraretinal fluid (SRF, IRF), which is indicative of persistent activity of the neovascular membrane. A pattern of dealing with such patients has not been established.

It is not clear to what extent the switch from one drug to another is effective for patients. Most of the studies reported that the switch from ranibizumab to intravitreal aflibercept is effective in improving retinal architecture [5-7]. In Poland; however, aflibercept may be the I line drug on a par with ranibizumab as part of the Drug Program for the Treatment of Age-Related Exudative Macular Degeneration (the Program). Thus, the patients who did not respond to this drug can be switched to ranibizumab. If no improvement is seen despite the change in medication, the switchback is available. We have taken the opportunity to investigate whether the switch from intravitreal aflibercept (IVA) to intravitreal ranibizumab (IVR) and than switchback to IVA show effectiveness in the management of non-responding patients, in whom further disease activity (the presence of any intra- and subretinal fluid) was observed after at least 12 months of primary treatment with aflibercept. Due to the nature of the study (retrospective analysis of documentation) and data anonymization, the consent of the Bioethics Committee was not necessary.

\section{MATERIAL}

We conducted a retrospective comparative case series study. Retrospective analysis covered the documentation and results of medical examinations of 20 eyes of 20 patients (male to female ratio $1: 1$; mean age: 75.8 years), treated in our Department from August 2015 to September 2020, who had no inhibition of disease activity (lack of dry retina due to persistent presence of IRF and/or SRF) after at least one year of aflibercept treatment, according to a rigid injection scheme bimonthly, after three initial injections every month. Criteria for inclusion in the Program include the presence of ac- tive (primary or secondary), classical, latent or mixed macular neovascularization (MNV) occupying more than 50\% of the changes in the course of AMD, confirmed by OCT and fluorescein angiography or angio-OCT examination; age over 45 years; lesion size less than 12 disc area (DA) BCVA in the treated eye 0.2-0.8 determined according to the Snellen chart (or ETDRS equivalent, respectively); the patient's consent to perform intravitreal injections; lack of predominant geographical atrophy; lack of dominant hemorrhage; lack of significant permanent damage to the foveal structure prior to treatment (significant structural damage is defined as present fibrosis or foveal atrophy or significant chronic discoid scar). The selection criteria must be met cumulatively.

We divided the study population into two groups: those who had not been treated with the anti-VEGF injections before starting treatment in the Program (treatment-naïve) ( $n=12 ; 60 \%)$, and those who had received injections before enrollment to the Program ( $\mathrm{n}=8 ; 40 \%)$. We switched patients to ranibizumab, due to lack of inhibition of disease activity (persistent presence of IRF and/or SRF). If the response to the drug was again inadequate, we returned to aflibercept after at least seven ranibizumab injections in the PRN (pro re nata) scheme. The number of at least seven injections after changing the drug results from the requirements of the Program. The mean number of all injections was $21.15 \pm 3.45 ; 12.95 \pm 2.80$ and $8.2 \pm 2.31$ IVA, IVR respectively. Visual acuity was tested on Snellen charts and then converted into the number of the Early Treatment Diabetic Retinopathy Study (ETDRS) letters. Optical coherence tomography (OCT) and retinal thickness (CRT) measurements were performed using OCT Triton (Topco, Japan). Best corrected visual acuity, CVA, CRT, IRF and SRF were assessed at the following points in the treatment process: on enrollment, after the third and seventh injections of aflibercept, before first, after third, and after the last injection of ranibizumab, and after the first injection of aflibercept after switchback.

\section{METHODS}

\section{Data analysis}

Data was analyzed using R 4.0.2 statistical software. To compare changes in parameters between measurements (e.g., between switching from IVA to IVR) and between groups, for each parameter a Bayesian multilevel regression was first conducted. Specifically, for SRF and IRF logistic regression was used, for BCVA transformed to 0-1 scale a zero-one inflated beta regression, and for log transformed CRT a linear regression. In Bayesian statistics the inference is based on analyzing posterior probability distributions of a model parameters, obtained by integrating likelihood (data) with 
prior probability distributions. The parameter (e.g., regression weight of group effect) is said to be statistically credible when $95 \%$ credible intervals (CI) of the posterior distribution exclude zero. As a point estimate of the effect medians of the posterior distributions are presented. As priors, standard normal non-informative distributions were used.

Patient group was coded with sum-to-zero contrast, and measurement effect with third order orthogonal polynomial (i.e., the contrast allowed for nonlinear patterns over the study course) to capture possibly nonlinear trends in parameter changes over measurements (as indicated by in exploratory analyses). Interaction term of measurement time and group was also included. The models were next used to compute posterior predicted marginal means, which are simply predicted mean values of a given parameter for a given measurement time and group. Posterior distributions of the means were next used to conduct planned pairwise comparisons: directly compare average values of parameters between groups on specific measurement times (e.g., at the end of the treatment). Note that these posterior distributions incorporate the uncertainty in estimates resulting from small sample sizes.

To approximated posterior distributions of the models Markov Chain Monte Carlo (MCMC) sampling procedure was conducted using brms package. For each reported model, six parallel MCMC chains were used. Each chain consisted of 6,000 samples, with 3,000 samples used as warm-up period and every $10^{\text {th }}$ sample recorded, resulting in 1,800 recorded samples in total. Sampling procedure was efficient and resulted with well-mixed and autocorrelation-free chains and unimodal posteriors.

\section{RESULTS}

\section{Changes in BCVA parameter}

Model parameters for the BCVA parameter are summarized in table 1 , while estimated marginal means and planned pairwise comparisons are presented in figure 1. We observed statistically credible interaction of the groups and linear trend of measurement, indicating that in the previously untreated group, the BCVA values increased reliably between consecutive measurements. In the previously treated group, the BCVA values did not change credibly over time (fig. 1, diagram on the right).

Results of the planned pairwise comparison (fig. 1, left diagram) indicated that in both groups differences between switch and reswitch measurements, and before and after IVR administration were not statistically credible. Average BCVA values between first and last injections and between first and last aflibercept administration (even before switching to ranibizumab) were credibly different only in the treatment-naïve group.

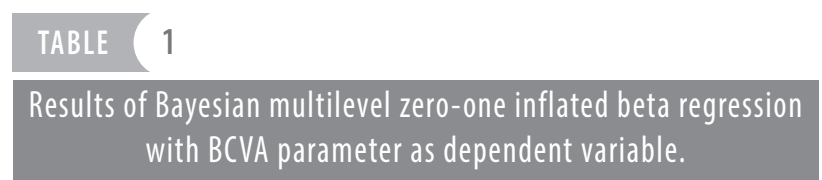

\begin{tabular}{|c|c|c|c|c|c|}
\hline \multicolumn{2}{|c|}{ Parameter } & \multirow{2}{*}{$\begin{array}{l}\text { Estimate } \\
1.6\end{array}$} & \multirow{2}{*}{$\begin{array}{l}\text { SE } \\
0.14\end{array}$} & \multirow{2}{*}{$\begin{array}{l}\text { LI } \\
1.31\end{array}$} & \multirow{2}{*}{$\begin{array}{l}\text { UI } \\
1.88\end{array}$} \\
\hline \multirow{8}{*}{$\beta$} & Intercept & & & & \\
\hline & Measurement [L] & 0.1 & 0.11 & -0.1 & 0.31 \\
\hline & Measurement [Q] & -0.07 & 0.1 & -0.26 & 0.13 \\
\hline & Measurement [C] & 0.04 & 0.1 & -0.15 & 0.24 \\
\hline & Group & 0.17 & 0.14 & -0.12 & 0.45 \\
\hline & Group : Measur. [L] & 0.27 & 0.1 & 0.06 & 0.46 \\
\hline & Group : Measur. [Q] & -0.01 & 0.1 & -0.21 & 0.19 \\
\hline & Group : Measur. [C] & 0.07 & 0.1 & -0.14 & 0.27 \\
\hline \multirow[t]{6}{*}{$\tau$} & Intercept & 0.58 & 0.12 & 0.4 & 0.84 \\
\hline & $\Phi$ & 36.57 & 4.88 & 27.77 & 46.79 \\
\hline & Zoi & 0.05 & 0.02 & 0.02 & 0.09 \\
\hline & Coi & 0.88 & 0.11 & 0.61 & 1 \\
\hline & $\mathrm{R}^{2}$ & 0.1 & 0.06 & 0.02 & 0.25 \\
\hline & $\mathrm{R}^{2}$ total & 0.55 & 0.05 & 0.45 & 0.63 \\
\hline
\end{tabular}

$\mathrm{L}, \mathrm{Q}$ and $\mathrm{C}$ - linear, quadratic and cubic trends, respectively; $\beta$ - regression weight; $\tau$ - standard deviation of individual intercepts (i.e., patient random effect); $\Phi$ - a shape parameter' zoi and coi - zero-one and conditional one inflation parameters.

Estimate is median of posteriori distribution, $\mathrm{SE}$ is standard error of the posterior, and $\mathrm{LI}$ and UI are lower and upper intervals of the $95 \%$ credible interval.

\section{Changes in CRT parameter}

Model parameters for the CRT (central retinal thickness) parameter are summarized in table 2, while estimated marginal means and planned pairwise comparisons are presented in figure 2. We did not observe any statistically credible effects in the model, but the overall model fit was adequate, as indicated by the $\mathrm{R}^{2}$ values. This indicates that the groups did not differ credibly in terms of change in macular central retinal thickness parameters over time

\section{TABLE 2}

Results of Bayesian multilevel linear regression with CRT parameter as dependent variable.

\begin{tabular}{|c|c|c|c|c|c|}
\hline \multicolumn{2}{|c|}{ Parameter } & \multirow{2}{*}{$\begin{array}{l}\text { Estimate } \\
-0.07\end{array}$} & \multirow{2}{*}{$\begin{array}{l}\text { SE } \\
0.17\end{array}$} & \multirow{2}{*}{\begin{tabular}{|l|} 
LI \\
-0.43
\end{tabular}} & \multirow{2}{*}{\begin{tabular}{|l} 
UI \\
0.28
\end{tabular}} \\
\hline \multirow{8}{*}{$\beta$} & Intercept & & & & \\
\hline & Measurement [L] & -0.14 & 0.15 & -0.41 & 0.15 \\
\hline & Measurement [Q] & 0.14 & 0.14 & -0.14 & 0.42 \\
\hline & Measurement [C] & -0.69 & 0.15 & -0.98 & -0.39 \\
\hline & Group & 0.33 & 0.18 & -0.03 & 0.68 \\
\hline & Group : Measur. [L] & -0.19 & 0.15 & -0.48 & 0.09 \\
\hline & Group : Measur. [Q] & 0.12 & 0.14 & -0.15 & 0.39 \\
\hline & Group : Measur. [C] & 0.12 & 0.15 & -0.17 & 0.4 \\
\hline \multirow[t]{4}{*}{$\tau$} & Intercept & 0.72 & 0.15 & 0.49 & 1.08 \\
\hline & $\Sigma$ & 0.65 & 0.04 & 0.57 & 0.74 \\
\hline & $\mathrm{R}^{2}$ & 0.21 & 0.08 & 0.07 & 0.37 \\
\hline & $\mathrm{R}^{2}$ total & 0.6 & 0.04 & 0.51 & 0.67 \\
\hline
\end{tabular}

L, Q and C - linear, quadratic and cubic trends, respectively; $\beta$ - regression weight; $\tau$ - standard deviation of individual intercepts (i.e., patient random effect); $\Sigma$ - a shape parameter.

parameter.
Estimate is median of posteriori distribution, $\mathrm{SE}$ is standard error of the posterior, and $\mathrm{LI}$ and UI are lower and upper intervals of the $95 \%$ credible interval. 
Pairwise comparisons (left) and posterior predicted marginal means (right) for the BCVA parameter. Single point is a median of a posterior distribution and horizontal (left) or vertical (right) lines are $95 \%$ credible intervals. Note that pairwise comparisons are on the logit scale. When the $95 \%$ credible interval does not include zero (i.e., does not cross the blue vertical line) the difference is statistically credible.
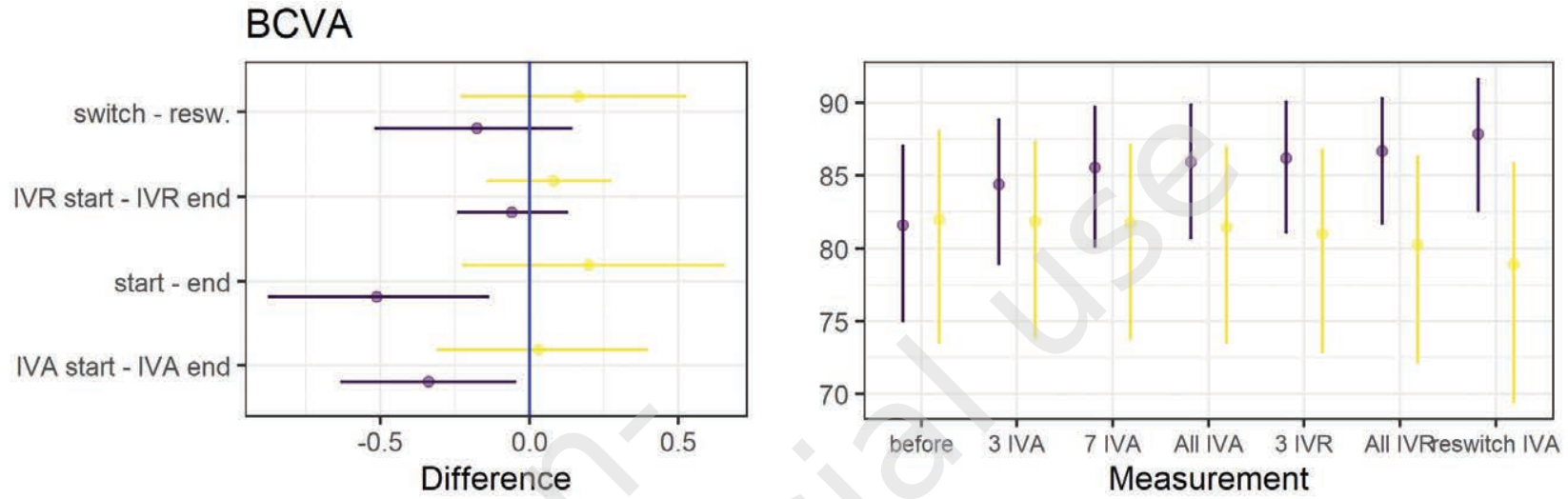

Treated before $\rightarrow$ no - yes

FIGURE (

Pairwise comparisons (left) and posterior predicted marginal means (right) for the CRT parameter. Single point is a median of a posterior distribution and horizontal (left) or vertical (right) lines are 95\% credible intervals. Note that pairwise comparisons are on a standard normal scale of log transformed CRT. When the $95 \%$ credible interval does not include zero (i.e., does not cross the blue vertical line) the difference is statistically credible.
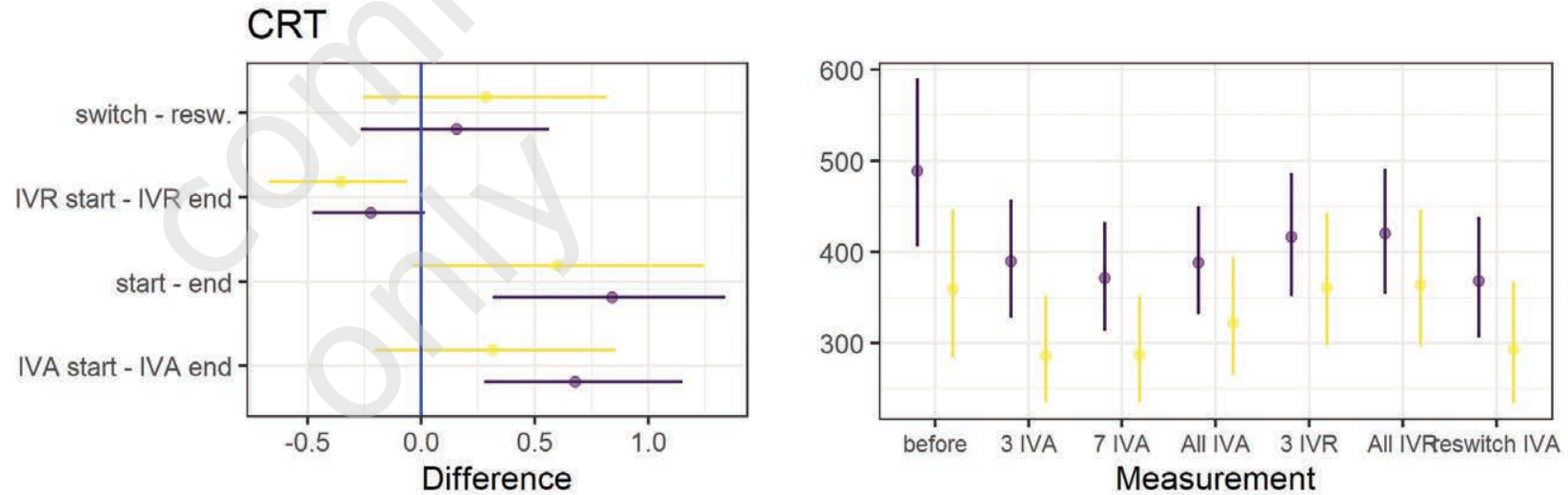

Treated before $\rightarrow$ no $\rightarrow$ yes

and also that no obvious trend in the parameter change was present as a result of the treatment (fig. 2, right diagram).

The results of the planned pairwise comparison (fig. 2, left diagram) indicated that switching to IVR in the treatment group resulted in a small, but statistically reliable increase in macular central retinal thickness. Additionally, in the previously untreated group, the mean CRT value decreased reliably between the first and last injections and between the first and last injections of IVA (before reswitch to ra- nibizumab). No such effects were observed for the previously treated group.

\section{Changes in the SRF and IRF parameters}

Model parameters for the SRF and IRF are summarized in table 3. We did not observe any statistically credible effects for the SRF (subretinal fluid) and IRF (intraretinal fluid) parameters. Furthermore, planned pairwise comparisons revealed no statistically credible differences between the compared predicted proportions. 


\section{DISCUSSION}

We focused on a group of patients that we termed "poorly responding to anti-VEGF therapy" because throughout the observation period, regardless of the drug used, we observed features of MNV activity in the form of sub- and/ or intraretinal fluid in macular OCT scans. Of course, the presence of MNV activity despite the treatment used is not unusual. Barthelmes et al. in their study showed $10 \%$ of active MNVs at the end of the second year of treatment, while Chandra et al. observed MNV activity in $1 / 3$ of patients after 10 years of treatment $[4,5]$.

The results of our work show that previously untreated patients achieve a credibly reliable improvement in VA at the end of the study, despite the persistence of disease activity. Posterior predicted means for BCVA in the treatment-naïe group, measured before treatment and at the end of the study were 81.58; 95\% CI: [74.95; 87.09] and 87.86, 95\% CI: [82.52; 91.7], respectively. Moreover, these patients gained the most after reswitch to aflibercept following ranibizumab administration $(86.65 ; 95 \%$ CI: $[81.62 ; 90.40])$. These results are consistent with the publications of other authors who indicated the positive impact of the lack of prior treatment on the functional effect of anti-VEGF therapy [6].

There is a clear lack of a credible global effect of the applied treatment on the thickness of the macular retina in both groups of patients (tab. 2). This is probably due to our selection of the study group in which disease activity was present all the time. Interestingly; however, there were two statistically credible differences in the subgroup of previously untreated patients in two periods (the same in which we observed changes in BCVA), namely a reliable reduction in macular retinal thickness during aflibercept therapy as well as after returning to aflibercept: $\Delta \mathrm{M}=0.68 ; 95 \% \mathrm{CI}$ : [0.28; 1.15], $\Delta \mathrm{M}=0.84 ; 95 \% \mathrm{CI}$ : $0.31 ; 1.34$ ], respectively (fig. 2 ).

There are more reports in the literature about switching from ranibizumab to aflibercept, or from bevacizumab to aflibercept or ranibizumab than the other way around [7-11]. The results of these studies vary: some show the effectiveness of the switch in the form of anatomical improvement (CRT) and functional improvement (BCVA) $[7,9,10]$; others show anatomical improvement, but no changes in BCVA $[8,10$, 11]. The SAFARI study, in which the authors assessed the effectiveness of the switch from aflibercept to ranibizumab, showed greater anatomical than functional improvement in the studied patients [12]. Our analyzes did not confirm the effectiveness of the switch from IVA to IVR in terms of any of the parameters tested (BCVA, CRT, SRF, IRF).

There is sparse data about the effectiveness of switchback to aflibercept. We are aware of the two studies that analyze this problem $[13,14]$. Koike et al. observed a decline in BCVA after switch, and improvement of both BCVA and CRT after switchback, which gives similar results to our observations [13]. However, the treatment regimen for both before (three initial injections of aflibercept and than the treat-and-extend regimen) and after switch (three injection of ranibizumab) was different than in our study. The scheme according to which our patients are treated is predefined by the requirements of the Program. Despreaux et al. also reported greater benefit the switchback than switch, both in BCVA and CRT, in patients initially treated with IVA [14].

The effectiveness of the switchback may be suggested by the phenomenon of tachyphylaxis. Tachyphylaxis is observed when dealing with a decreased response to the drug with long-term use [13, 15]. Questions to be asked: if we can consider the phenomenon of tachyphylaxis and the switch did not improve either functional or anatomical, would it be a better choice to suspend the therapy? Although it takes a lot of courage for the physician to introduce a drug holiday for a patient with a persistent neovascular membrane activity, it seems to be a reasonable socioeconomic choice. An aspect that is rarely discussed in the literature is futility of treatment [16]. Although statistical analysis confirmed the treatment efficacy, it is still unclear how to deal with poorly responding patients, how long should patients be treated, and what should be a predictor of treatment discontinuation. Treatment of this disease entity covers seniors, often burdened with additional general diseases, for whom the chronicity of therapy is subjectively more burdensome than the measurable treatment benefits [17].

Despite the statistically noticeable improvement in the BCVA and CRT parameters, we were unable to achieve a dry retina throughout the treatment period (tab. 3). The question arises as to may the effectiveness of the therapy can be assessed if the improvement in BCVA is accompanied by sustained MNV activity. However, what we perceive a limitation of the study is the fact that the IRF/SRF was analyzed qualitatively rather than quantitatively, and we did not evaluate the change in IRF/SRF quantity during treatment, which might better indicate a tissue response to the drug.

The main limitation of the study was the small number of patients. However, by using Bayesian multilevel models and probability distributions carefully matched to the type of dependent, we were able to reliably estimate posteriors of parameter values and use them for inference. These estimates naturally control individual differences and small sample sizes by adjusting, which is reflected in the width of a credible interval.

The results of our work do not sufficiently answer the question of how to manage a patient poorly responding to aflibercept treatment. More research is needed in this area.

\section{CONCLUSIONS}

The study showed that treatment conversion may be beneficial for patients poorly responding to primary therapy. Our 
TABLE 3

\begin{tabular}{|c|c|c|c|c|c|c|c|c|c|}
\hline \multirow{2}{*}{\multicolumn{2}{|c|}{$\begin{array}{l}\text { Dependent variable } \\
\text { Parameter }\end{array}$}} & \multicolumn{4}{|c|}{ SRF } & \multicolumn{4}{|c|}{ IRF } \\
\hline & & Estimate & SE & $\mathrm{LI}$ & UI & Estimate & SE & $\mathrm{LI}$ & UI \\
\hline \multirow{8}{*}{$\beta$} & Intercept & 2.55 & 0.4 & 1.8 & 3.42 & -1.75 & 0.63 & -2.97 & -0.51 \\
\hline & Measurement [L] & -0.91 & 0.6 & -2.14 & 0.27 & -0.09 & 0.6 & -1.24 & 1.14 \\
\hline & Measurement [Q] & -1 & 0.67 & -2.37 & 0.26 & 0.66 & 0.6 & -0.49 & 1.88 \\
\hline & Measurement [C] & -0.75 & 0.67 & -1.99 & 0.57 & -0.03 & 0.61 & -1.23 & 1.15 \\
\hline & Group & 0.02 & 0.4 & -0.8 & 0.84 & 0.06 & 0.62 & -1.2 & 1.29 \\
\hline & Group : Measurement [L] & -0.08 & 0.63 & -1.25 & 1.18 & 0.45 & 0.6 & -0.69 & 1.64 \\
\hline & Group : Measurement [Q] & 0.71 & 0.65 & -0.53 & 2.02 & -0.74 & 0.6 & -1.91 & 0.38 \\
\hline & Group : Measurement [C] & -0.38 & 0.66 & -1.69 & 0.94 & 0.23 & 0.63 & -1.02 & 1.49 \\
\hline$\tau$ & Intercept & 0.65 & 0.52 & 0.02 & 1.93 & 2.86 & 1.04 & 1.41 & 5.38 \\
\hline
\end{tabular}

$\mathrm{L}, \mathrm{Q}$ and $\mathrm{C}$ - linear, quadratic and cubic trends, respectively; $\beta$ - regression weight; $\tau$ - standard deviation of individual intercepts (i.e., patient random effect).

Estimate is median of posteriori distribution, SE is standard error of the posterior, and LI and UI are lower and upper intervals of the $95 \%$ credible interval.

work shows that anatomical success does not always occur with functional success. Despite persistent disease activity, intensive anti-VEGF treatment is necessary not only to stabilize, but also to improve BCVA. Treatment-naïve patients achieve most from this regimen in terms of improved VA and decreased CRT.

Figures: from the author's own materials.

\section{CORRESPONDENCE}

Małgorzata Wichrowska, MD

Department of Ophthalmology,

Poznan University of Medical Sciences

60-569 Poznań, ul. A. Szamarzewskiego 84

e-mail:wichrovska@gmail.com

\section{ORCID}

Małgorzata Wichrowska - ID - http://orcid.org/0000-0002-1523-6222 Jarosław Kocięcki - ID - http://orcid.org/0000-0001-7321-1835 Wojciech Suda - ID - http://orcid.org/0000-0002-7977-8274

\section{References}

1. Supuran CT. Agents for the prevention and treatment of age-related macular degeneration and macular edema: a literature and patent review. Expert Opin Ther Pat. 2019; 29(10): 761-7. http://doi.org/10.1080/13543776.2019.1671353.

2. Yazdi MH, Faramarzi MA, Nikfar S et al. Ranibizumab and aflibercept for the treatment of wet age-related macular degeneration. Expert Opin Biol Ther. 2015; 15(9): 1349-58. http://doi.org/10.1517/14712598.2015.1057565.

3. Smit C, Wiertz-Arts K, van de Garde EM. Intravitreal aflibercept versus intravitreal ranibizumab in patients with age-related macular degeneration: a comparative effectiveness study. J Comp Eff Res. 2018; 7(6): 561-7. http://doi.org/10.2217/cer-2017-0099.

4. Chandra S, Arpa C, Menon D et al. Ten-year outcomes of antivascular endothelial growth factor therapy in neovascular age-related macular degeneration. Eye (Lond). 2020; 34(10): 1888-96. http://doi.org/10.1038/s41433-020-0764-9.

5. Barthelmes D, Nguyen V, Daien V et al; Fight Retinal Blindness Study Group. Two year outcomes of "treat and extend" intravitreal therapy using aflibercept preferentially for neovascular age-related macular degeneration. Retina. 2018; 38(1): 20-8. http://doi.org/10.1097/ IAE.0000000000001496.

6. Wachtlin J, Eter N, Hasanbasic Z et al. Importance of continuous treatment with intravitreal aflibercept injections in patients with neovascular age-related macular degeneration-12-month post hoc analysis of the PERSEUS real-world evidence study. Graefes Arch Clin Exp Ophthalmol. 2021; 259(3): 601-11. http://doi.org/10.1007/s00417-020-04803-8. Epub 2020.

7. Salcedo-Villanueva G, Feria-Anzaldo E, Romo-Aguas JC et al. Anti-VEGF treatment switch in neovascular age-related macular degeneration: a comparison of aflibercept versus ranibizumab after a single-dose switch. Int Ophthalmol. 2019; 39: 2023-31. http://doi. org/10.1007/s10792-018-1038-4. 
8. Chakravarthy U, Bezlyak V, Sagkriotis A et al. Effectiveness of Continued Ranibizumab Therapy in Neovascular Age-Related Macular Degeneration versus Switch to Aflibercept: Real World Evidence Presented at: 17th EURETINA Congress, September 2017, Barcelona, Spain. http://doi.org/10.1016/j.oret.2018.09.001.

9. Spooner K, Hong T, Nair R et al. Long-term outcomes of switching to aflibercept for treatment-resistant neovascular age-related macular degeneration. Acta Ophthalmol. 2019; 97(5): e706-e12. http://doi.org/110.1111/aos.14046.

10. de Sá Quirino-Makarczyk L, Sainz Ugarte MF, Viana Vieira B et al. Short-term results of early switch from Ranibizumab to Aflibercept in poor or non responder age related macular degeneration in clinical practice. Int J Retina Vitreous. 2020; 6: 9. http://doi.org/10.1186/ s40942-020-00212-5.

11. Ertan E, Doğan M, Polat $O$ et al. Switch to aflibercept in the treatment of neovascular age-related macular degeneration: $30-$ month results. Arq Bras Oftalmol. 2021. http://doi.org/10.5935/0004-2749.20210036.

12. Gale RP, Pearce I, Eter $\mathrm{N}$ et al. Anatomical and functional outcomes following switching from aflibercept to ranibizumab in neovascular age-related macular degeneration in Europe: SAFARI study. Br J Ophthalmol. 2020; 104(4): 493-9. http://doi.org/10.1136/bjophthalmol-2019-314251.

13. Koike N, Otsuji T, Tsumura A et al. Results of switchback from ranibizumab to aflibercept in patients with exudative age-related macular degeneration. Clin Ophthalmol. 2019; 13: 1247-51. http://doi.org/10.2147/OPTH.S206910.

14. Despreaux R, Cohen SY, Semoun O et al. Short-term results of switchback from aflibercept to ranibizumab in neovascular age-related macular degeneration in clinical practice. Graefes Arch Clin Exp Ophthalmol. 2016; 254(4): 639-44. http://doi.org/10.1007/s00417-015-3084-1.

15. Hara C, Wakabayashi T, Fukushima Y et al. Tachyphylaxis during treatment of exudative age-related macular degeneration with aflibercept. Graefes Arch Clin Exp Ophthalmol. 2019; 257(11): 2559-69. http://doi.org/10.1007/s00417-019-04456-2.

16. Justin GA, Rapuano CJ, Pelton RW et al. Ocular Futility: How to Approach End-of-Sight Care. Ophthalmology. 2019; 126(9): $1209-11$. http://doi.org/10.1016/j.ophtha.2019.06.027.

17. Senra H, Balaskas K, Mahmoodi N et al. Experience of Anti-VEGF Treatment and Clinical Levels of Depression and Anxiety in Patients With Wet Age-Related Macular Degeneration. Am J Ophthalmol. 2017; 177: 213-24. http://doi.org/10.1016/j.ajo.2017.03.005.

Authors' contributions:

All authors contributed equally to the article.

Conflict of interest:

None.

Financial support:

None.

Ethics:

The content presented in the article complies with the principles of the Helsinki

Declaration, EU directives and harmonized requirements for biomedical journals. 